\title{
Brillouin zone center phonon modes in $\mathrm{ZnGa2O4}$
}

Megan Stokey, Rafal Korlacki, Sean Knight, Matthew Hilfiker, Zbigniew Galazka, Klaus Irmscher, Vanya Darakchieva and Mathias Schubert

The self-archived postprint version of this journal article is available at Linköping University Institutional Repository (DiVA):

http://urn.kb.se/resolve?urn=urn:nbn:se:liu:diva-169240

N.B.: When citing this work, cite the original publication.

Stokey, M., Korlacki, R., Knight, S., Hilfiker, M., Galazka, Z., Irmscher, K., Darakchieva, V., Schubert, M., (2020), Brillouin zone center phonon modes in $\mathrm{ZnGa2O}$, Applied Physics Letters, 117(5), 052104. https://doi.org/10.1063/5.0012526

Original publication available at:

https://doi.org/10.1063/5.0012526

Copyright: American Institute of Physics

http://www.aip.org/ 


\title{
Brillouin zone center phonon modes in $\mathrm{ZnGa}_{2} \mathrm{O}_{4}$
}

\author{
Megan Stokey, ${ }^{1, \text { a) }}$ Rafał Korlacki, ${ }^{1}$ Sean Knight, ${ }^{1}$ Matthew Hilfiker, ${ }^{1}$ Zbigniew Galazka, ${ }^{2}$ Klaus Irmscher, ${ }^{2}$ \\ Vanya Darakchieva, ${ }^{3}$ and Mathias Schubert ${ }^{1,3,4}$ \\ ${ }^{1)}$ Department of Electrical and Computer Engineering, University of Nebraska-Lincoln, Lincoln, NE 68588, \\ USA \\ ${ }^{2)}$ Leibniz-Institut für Kristallzüchtung, 12489 Berlin, Germany \\ ${ }^{3)}$ Terahertz Materials Analysis Center and Center for III-N Technology, C3NiT - Janzèn, \\ Department of Physics, Chemistry and Biology (IFM), Linköping University, 58183 Linköping, \\ Sweden \\ ${ }^{4)}$ Leibniz Institut für Polymerforschung e.V., 01069 Dresden, Germany \\ (Dated: 17 July 2020)
}

Infrared-active lattice mode properties in melt grown high quality single bulk crystals of $\mathrm{ZnGa}_{2} \mathrm{O}_{4}$ are investigated by a combined spectroscopic ellipsometry and density functional theory computation analysis. The normal spinel structure crystals are measured by spectroscopic ellipsometry at room temperature in the range of $100 \mathrm{~cm}^{-1}-1200 \mathrm{~cm}^{-1}$. The complex-valued dielectric function is determined from a wavenumber-by-wavenumber approach, which is then analyzed by the four-parameter semi-quantum model dielectric function approach augmented by impurity mode contributions. We determine four infrared-active transverse and longitudinal optical mode pairs, five localized impurity mode pairs, and the high frequency dielectric constant. All four infrared-active transverse and longitudinal optical mode pairs are in excellent agreement with results from our density functional theory computations. With the LyddaneSachs-Teller relationship we determine the static dielectric constant, which agrees well with electrical capacitance measurements performed on similarly grown samples. We also provide calculated parameters for all Raman-active and for all silent modes, and thereby provide a complete set of all symmetry predicted Brillouin zone center modes.

Transparent conductive oxides have been the focus of many research efforts due to their wide range of physical properties which make them suitable, for example, for a large variety of electronic and optoelectronic device applications. ${ }^{1-3}$ Divalent metal (Me) based ternary Ga-based spinels, $\mathrm{MeGa}_{2} \mathrm{O}_{4}$, have recently regained interest due to their potential for grow in high quality single-crystal phases, and due to their potential for useful ultrawide band gap semiconductor properties. ${ }^{4,5}$ $\mathrm{ZnGa}_{2} \mathrm{O}_{4}$ (zinc gallate; $\mathrm{ZGO}$ ) is one of such material with a band gap energy of $4.59 \pm 0.03 \mathrm{eV}$ assuming a direct optical transition (or of about $4.3 \mathrm{eV}$ assuming an indirect optical transition) reported recently by Galazka et al. ${ }^{4}$ The ultrawide band gap semiconductor $\beta-\mathrm{Ga}_{2} \mathrm{O}_{3}$ (gallium sesquioxide) has similar large band gap energy values which strongly depend on the polarization direction within the highly anisotropic crystal structure. ${ }^{4,6,7}$ This monoclinic symmetry phase of gallium oxide is a contemporary material of interest with high promises for use in high-power, high-voltage applications. ${ }^{8-11}$ Where $\beta-\mathrm{Ga}_{2} \mathrm{O}_{3}$ continues to be a difficult material to work with due to its crystallographic monoclinic symmetry, $\mathrm{ZnGa}_{2} \mathrm{O}_{4}$ offers a promising high-symmetry alternative. Galazka et al. recently demonstrated controllable electrical conductivity in bulk $\mathrm{ZnGa}_{2} \mathrm{O}_{4}$ single crystals including highly electrically insulating material. ${ }^{4}$ For example, a Hall mobility of about $100 \mathrm{~cm}^{2} / \mathrm{Vs}$ at a free electron concentration of approximately $1 \times 10^{19} \mathrm{~cm}^{-3}$ was reported. Such high Hall mobility for such large free electron concentration is currently unavailable in single crystal substrates of $\beta-\mathrm{Ga}_{2} \mathrm{O}_{3}$. Good electrical mobility at high carrier concentration can make bulk single crystal ZGOsuitable for applications in future vertical configuration high-power high-voltage devices. ${ }^{12}$

Presently, very little is known about fundamental physical properties of ZGO. Transition metal ion doped ZGO has been reported as long-lasting high brightness phosphor for in vivo imaging using $\mathrm{x}$-ray phosphorescence and thermally stimu-

a)Electronic mail: mstokey@ huskers.unl.edu; http://ellipsometry.unl.edu lated luminescence. ${ }^{13}$ Chemical and thermal stability make it suitable for use in plasma and field emission devices. ${ }^{14}$ Yang et al. reported synthesis of ZGO as multimetallic mesoporous materials with improved photocatalytic activity for $\mathrm{CO}_{2}$ reduction. ${ }^{15}$ While ZGO has been grown and researched as a single crystal grown from flux ${ }^{16-18}$ since the late 1960 's, most previously published work focused on thin films, ceramics, and polycrystalline samples..$^{13,19-22}$ Phonon modes are of basic interest for understanding carrier and heat transport, or photo excitation processes, for example. While some information on infrared (IR)-active as well as Raman-active modes was reported, a complete set of IR-active transverse (TO) and longitudinal optical (LO) phonon modes in ZGO is not available yet. ${ }^{23-27}$ Preudhomme et al. ${ }^{28}$ provided an early IR study of vibrational modes in II-III spinels. López et al. ${ }^{27}$ and López-Moreno et al. ${ }^{29}$ reported Raman-active modes, IRactive TO modes, and silent modes from density functional theory (DFT) calculations. López et al..$^{27}$ and Van Gorkom et $a l^{24}$ reported experimental Raman scattering results without identifying all Raman-active modes. In this work we provide calculated parameters for all Brillouin zone center phonon modes from DFT calculations, and we report the IR-active TO and LO phonon modes, obtained from spectroscopic ellipsometry analysis, in melt-grown high-quality bulk single crystal ZGO. We find all predicted IR-active modes in excellent agreement with our DFT calculations, and we report on the observation of additional, potentially impurity-related modes.

ZGO single crystals were obtained directly from the melt by the vertical gradient freeze method (VGF), as described in detail by Galazka et al. ${ }^{4}$ The obtained crystals were sliced with surfaces of (100) and (111) orientations, annealed at $1100^{\circ} \mathrm{C}$ in air for $10 \mathrm{~h}$, cut into wafers of $10 \times 10 \times 0.5 \mathrm{~mm}^{3}$, lapped, and one-side polished. As reported in Ref. 4, the sample growth conditions lead to electrically insulating crystals by annealing in the presence of oxygen at temperatures $\leq 700^{\circ} \mathrm{C}$ for at least several hours. Hence, our samples were colorless and electrically insulating. Samples were measured as received without further treatment. Radio frequency (RF) ca- 


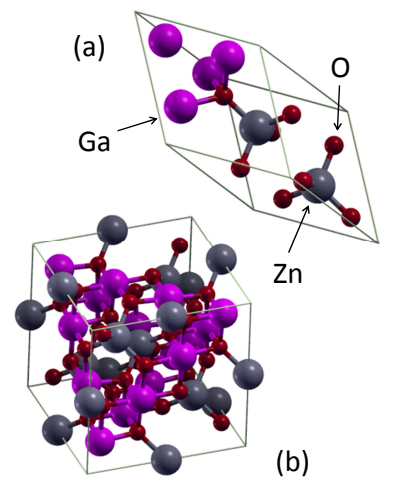

FIG. 1. Primitive (a) and conventional (b) cells of spinel structure ZGO crystal. Note that both cells have the same orientation with respect to an external coordinate system.

pacitance measurements (test frequency $1 \mathrm{kHz} \ldots 1 \mathrm{MHz}$, test level $100 \mathrm{mV}$ ) were performed on a capacitor geometry with $7 \mathrm{~mm}$ diameter gold contacts electron-beam evaporated on a ZGO wafer polished on both sides.

ZGO crystallizes in the cubic spinel structure (space group 227, F-3dm, Figure 1). There are 14 atoms ( 2 chemical units) in the primitive cell, which produce 42 phonon modes, of which three with $T_{1 \mathrm{u}}$ symmetry are acoustic modes and the $\Gamma$-point optical modes are classified as follows:

$\Gamma_{\mathrm{opt}}=A_{1 \mathrm{~g}}+2 A_{2 \mathrm{u}}+2 E_{\mathrm{u}}+E_{\mathrm{g}}+4 T_{1 \mathrm{u}}+2 T_{2 \mathrm{u}}+T_{1 \mathrm{~g}}+3 T_{2 \mathrm{~g}}$.

All $T$-symmetries modes are triple-degenerate and $E$ symmetries modes are double degenerate, for a total of 39 optical modes at the Brillouin zone center. $A_{1 \mathrm{~g}}, E_{\mathrm{g}}$, and $T_{2 \mathrm{~g}}$ modes are Raman-active. The IR-active modes $T_{1 \mathrm{u}}$ split into TO and LO modes. All other modes are silent.

Spectroscopic ellipsometry is a contact-less measurement technique which measures the change in polarization of light $(\tilde{\rho})$ caused by interaction with the sample. In reflection ellipsometry this change can be written as

$$
\tilde{\rho}=\frac{\tilde{r}_{p}}{\tilde{r}_{s}}=\tan (\Psi) e^{i \Delta}
$$

where $\tilde{r}_{p}$ and $\tilde{r}_{s}$ are the Fresnel reflection coefficients for the light polarized parallel to $(p)$ and perpendicular $(s)$ to the plane of incidence. The rotation of the light's polarization state about the axis of propagation is defined as $\Psi$ and the relative phase shift between the parallel and perpendicular components is defined as $\Delta$. Hence, for each wavenumber a ( $\Psi$, $\Delta$ ) pair is measured.

Two instruments were used to obtain the ellipsometric data. Mid-infrared data was taken from $500 \mathrm{~cm}^{-1}-1200 \mathrm{~cm}^{-1}$ using a commercially available variable angle of incidence spectroscopic ellipsometer (IR-VASE Mark-II; J.A. Woollam Co., Inc.). Far-infrared ellipsometry was performed from $100 \mathrm{~cm}^{-1}-500 \mathrm{~cm}^{-1}$ with an in-house built FIR-VASE ellipsometer. ${ }^{30}$ Data were acquired at $\Phi_{a}=50^{\circ}$ and $70^{\circ}$ angle of incidence. Measurements were repeated at multiple sample azimuths and found identical as expected due to the isotropic optical properties of cubic spinel structure. Measurements
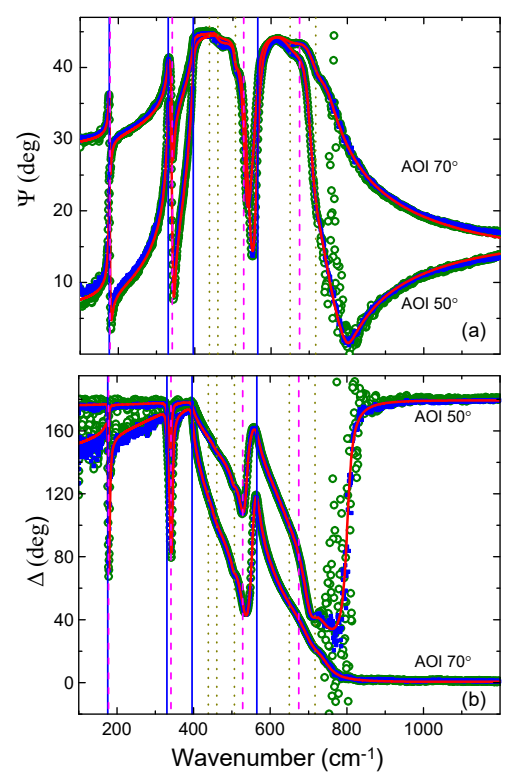

FIG. 2. Experimental (green open symbols), wavenumber-bywavenumber best-match model (blue closed symbols), and bestmatch dielectric function model (red solid line) calculated $\Psi$ (a), and $\Delta(b)$. Vertical blue solid lines indicate TO modes, magenta dashed lines indicate LO modes, and gold dotted lines indicate impurity modes identified in this work.

were performed on two different samples, one of each with (100) and (111) surface orientations, respectively. All measurements were done in stable, ambient conditions at room temperature. During analysis, data from both samples were combined and analyzed simultaneously. We note that data sets from different samples are identical to within the noise. Analysis was performed using WVASE32 ${ }^{\text {TM }}$ (J.A. Woollam Co., Inc.). Experimental data are shown in Fig. 2. We use the half-infinite ambient-substrate approximation for model analysis. No surface roughness is considered because the samples are highly polished, and residual nanometer-scale roughness does not contribute to the ellipsometric data in the mid-infrared and far-infrared regions. ${ }^{31}$ We first analyse the experimental data using a wavenumber-by-wavenumber best-match model calculation searching for the values of the real and imaginary parts of the substrate dielectric function. In this process, all parameters, the wavenumber dependent dielectric function values, are searched for independently, i.e., without any specific model lineshape assumption. The resulting best-match model-calculated ellipsometry data are shown in Fig. 2. Then, the wavenumber-by-wavenumber obtained dielectric function was analyzed using the so-called four-parameter semi-quantum (FPSQ) model first described by Gervais and Periou. ${ }^{32}$ This model permits direct access to TO and LO mode frequencies, $\omega_{\mathrm{TO}, l}$ and $\omega_{\mathrm{LO}, l}$, and allows for determination of their independent broadening parameters, 


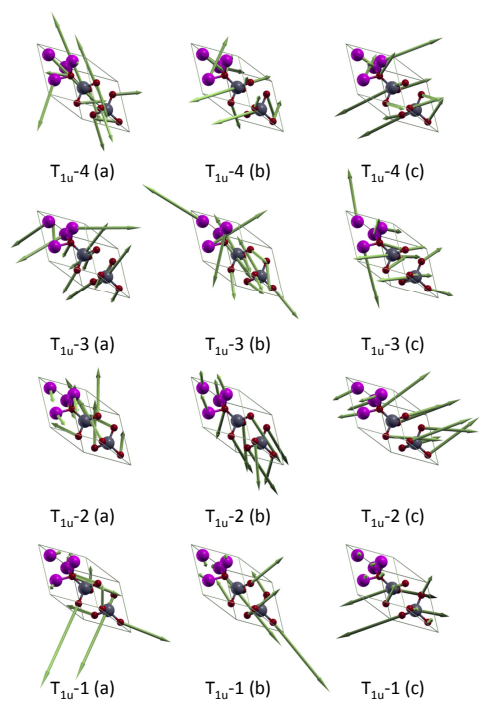

FIG. 3. Atomic displacement eigenvectors for IR-active phonon modes in the spinel structure single bulk crystal of ZGO. Because $T_{1 \mathrm{u}}$ modes are triple-degenerate, three eigenvectors labeled (a), (b), (c), are shown for each phonon mode. Note that the transition dipoles are orthogonal to each other for all triplets. Color scheme: grey - Zn, purple - Ga, red - O.

$\gamma_{\mathrm{TO}, l}$ and $\gamma_{\mathrm{LO}, l}$, respectively

$$
\varepsilon=\varepsilon_{\infty} \prod_{l=1}^{N+M} \frac{\omega_{\mathrm{LO}, l}^{2}-\omega^{2}-i \omega \gamma_{\mathrm{LO}, l}}{\omega_{\mathrm{TO}, l}^{2}-\omega^{2}-i \omega \gamma_{\mathrm{TO}, l}}
$$

and $\varepsilon_{\infty}$ is the high-frequency dielectric constant. ${ }^{31}$ The product runs over all $N=4$ phonons (TO-LO pairs) with $T_{1 \mathrm{u}}$ symmetry. In addition, as will be discussed further below, we identify $M=5$ impurity modes (I-1, ., I-5) with small polarity, i.e., with $\omega_{\mathrm{LO}, l}^{2}-\omega_{\mathrm{TO}, l}^{2} \approx 0 .^{33,34}$ The Lyddane-SachsTeller (LST) relation ${ }^{35}$ then permits calculation of the static dielectric constant, $\varepsilon_{\mathrm{DC}}$,

$$
\frac{\varepsilon_{\mathrm{DC}}}{\varepsilon_{\infty}}=\prod_{l=1}^{N+M} \frac{\omega_{\mathrm{LO}, l}^{2}}{\omega_{\mathrm{TO}, l}^{2}},
$$

using all other parameters in Eq. 2 determined from analysis of the wavenumber-by-wavenumber obtained $\varepsilon$.

Theoretical calculations were performed by plane wave DFT code Quantum ESPRESSO (QE) ${ }^{36}$ We used the local density approximation exchange-correlation functional of Perdew and Wang $(\mathrm{PW})^{37,38}$ and optimized norm-conserving Vanderbilt (ONCV) scalar-relativistic pseudopotentials. ${ }^{39}$ As the starting point we used structural parameters from the Materials Project. ${ }^{40,41}$ The initial structure was first relaxed to force levels less than $10^{-6} \mathrm{Ry} \mathrm{Bohr}^{-1}$. A regular shifted $4 \times 4 \times 4$ Monkhorst-Pack grid was used for sampling of the Brillouin zone. ${ }^{42}$ A convergence threshold of $1 \times 10^{-12}$

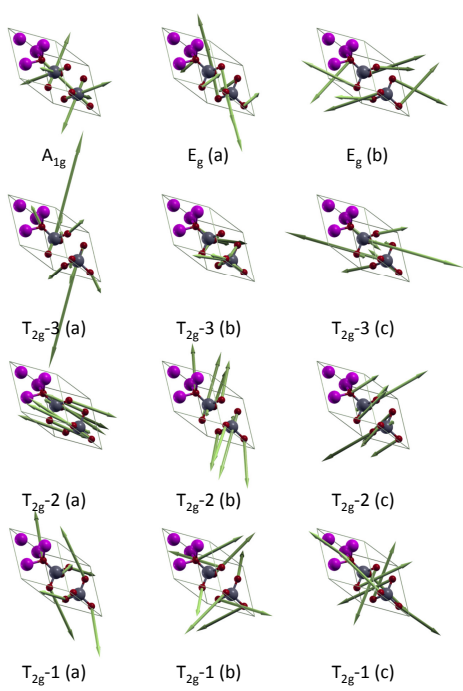

FIG. 4. Atomic displacement eigenvectors for Raman-active phonon modes in the spinel structure single bulk crystal of ZGO. Because $E_{8}$ modes are double-degenerate and $T_{1 \mathrm{u}}$ modes are triple-degenerate, two and three eigenvectors, respectively, labeled (a), (b), (c), are shown for these modes.

Ry was used to reach self consistency with a large electronic wavefunction cutoff of 200 Ry. The relaxed cell was used for subsequent phonon calculations. The phonon frequencies, Born effective charges, IR intensities, and Raman scattering activities ${ }^{43}$ were computed at the $\Gamma$-point of the Brillouin zone using density functional perturbation theory, ${ }^{44}$ as implemented in the Quantum ESPRESSO package, with the convergence threshold for self-consistency of $1 \times 10^{-18} \mathrm{Ry}$. The parameters of the TO modes were obtained from the dynamical matrix computed at the $\Gamma$-point. The parameters of the LO modes were obtained by setting a small displacement from the $\Gamma$-point in order to include the long-range Coulomb interactions of Born effective charges in the dynamical matrix (the so called non-analytical terms). We note that DFT calculations were performed disregarding effects of finite temperature.

Our DFT analysis results are summarized in Tab. I for all IR-active and all Raman modes, and in Fig. 5 for all silent modes. Renderings of the eigenvectors (the atomic displacement patterns, normalized to an arbitrary magnitude in order to visualize together modes with diverse oscillator strengths), prepared using XCrysDen ${ }^{45}$ running under Silicon Graphics Irix 6.5, are presented in Fig. 3 (IR-active modes), Fig. 4 (Raman-active modes), and Fig. 5 (silent modes). Note the diversity of the participating constituents and the complexity of their respective displacement pattern for all modes, where a simple subdivision into vibrations within or among sub-blocks within the spinel structure cannot be upheld. Our results for the IR-active TO modes frequencies, as well as for the Raman- 

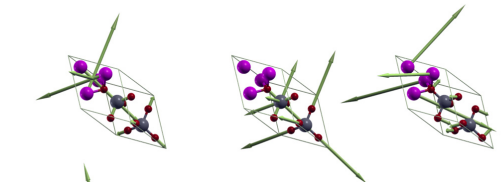

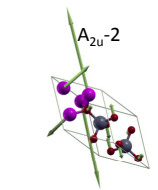

$\mathrm{A}_{2 u^{-1}}$

$\mathrm{E}_{\mathrm{u}}-2(\mathrm{a})$
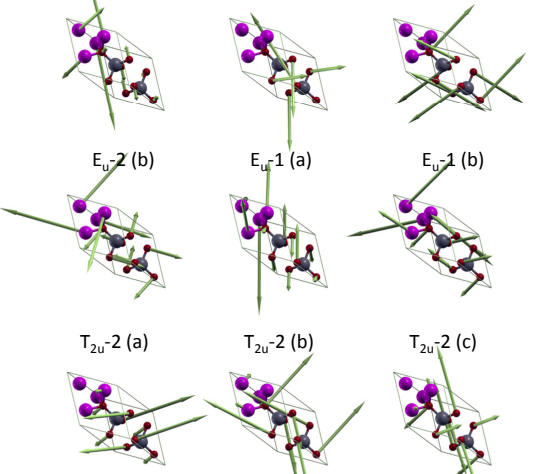

$\mathrm{T}_{2 \mathrm{u}}-2$ (b)
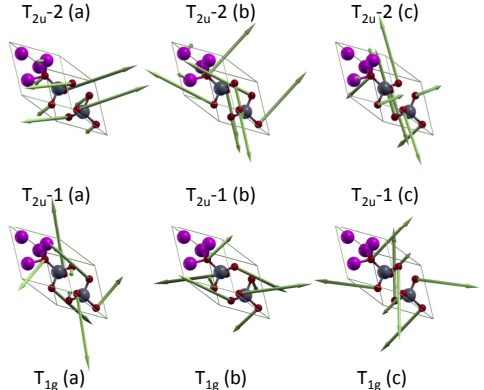

$\mathrm{T}_{2 \mathrm{u}}-1$ (c)

FIG. 5. Atomic displacement eigenvectors for silent phonon modes in the spinel structure single bulk crystal of ZGO. DFT-calculated frequencies of these modes are (numbers in brackets from Refs. 27 and 29)

: 403.20 (419) $\mathrm{cm}^{-1}\left(A_{2 \mathrm{u}}-2\right), 694.57(702) \mathrm{cm}^{-1}\left(A_{2 \mathrm{u}}-1\right), 218.65$ (229) $\mathrm{cm}^{-1}\left(E_{\mathrm{u}}-2\right), 547.04(563) \mathrm{cm}^{-1}\left(E_{1}-1\right), 130.14(135) \mathrm{cm}^{-1}$ $\left(T_{2 \mathrm{u}}-2\right), 422.09(450) \mathrm{cm}^{-1}\left(T_{2 \mathrm{u}}-1\right), 335.56(366) \mathrm{cm}^{-1}\left(T_{1 \mathrm{~g}}\right)$. Because $E_{\mathrm{u}}$ modes are double-degenerate and $T_{2 \mathrm{u}}$ and $T_{1 \mathrm{~g}}$ modes are triple-degenerate, two and three eigenvectors, respectively, labeled (a), (b), (c), are shown for these modes.

active and silent modes are consistent with the DFT-calculated phonon modes frequencies reported by López et al. ${ }^{27}$ and López-Moreno et al. (Tab. I). ${ }^{29}$ Calculated LO modes have not been reported yet and neither were the intensities of the IR-active modes or activities of the Raman-active modes. The latter are very small for the $\mathrm{E}_{\mathrm{g}}$ and $\mathrm{T}_{2 \mathrm{~g}}-3$ modes, which may explain why these were not detected in the experiments in Refs. 24 and 27

Our spectroscopic ellipsometry analysis identified four IRactive TO-LO mode pairs as predicted by DFT. These modes, all with $\mathrm{T}_{1 \mathrm{u}}$ symmetry, can be clearly seen in the dielectric function, which is shown in Fig. 6. The best-match model dielectric function parameters are listed in Tab. II. The TO mode frequencies are mostly in agreement with those reported by Van Gorkom et al. ${ }^{24}$ and Preudhomme et al. ${ }^{28}$ also listed in Tab. II. LO mode frequencies were not reported from experiment so far. Our mode frequency parameters determined from
TABLE I. Parameters for IR-active and Raman-active phonon modes obtained from DFT calculations in this work, and compared with literature data where available

Frequencies, $\omega_{\mathrm{TO}}$ and $\omega_{\mathrm{LO}}$, and IR intensities, $A_{\mathrm{TO}}^{2}$ and $A_{\mathrm{LO}}^{2}$ respectively, of TO and $\mathrm{LO}$ modes are presented for IR-active modes. Frequencies, $\omega_{\mathrm{RA}}$, and Raman scattering activities, $S_{\mathrm{RA}}$, are provided for Raman-active modes. Frequencies for silent modes are shown in Fig. 5. Values for $\varepsilon_{\infty}$ and $\varepsilon_{\mathrm{DC}}$ are determined as 4.128 , and 10.63 , respectively, using the LST relationship.

\begin{tabular}{|c|c|c|c|c|c|}
\hline Mode & $\begin{array}{c}\omega_{\mathrm{TO}} \\
\left(\mathrm{cm}^{-1}\right)\end{array}$ & $\begin{array}{c}\omega_{\mathrm{TO}} \\
\left(\mathrm{cm}^{-1}\right)\end{array}$ & $\begin{array}{c}\omega_{\mathrm{LO}} \\
\left(\mathrm{cm}^{-1}\right)\end{array}$ & $\begin{array}{c}A_{\mathrm{TO}}^{2} \\
\left(\frac{(\mathrm{D} / \mathrm{A})^{2}}{\mathrm{amy}}\right)\end{array}$ & $\begin{array}{c}A_{\mathrm{LO}}^{2} \\
\left(\frac{(\mathrm{D} / \AA)^{2}}{3 m y}\right)\end{array}$ \\
\hline$\overline{T_{1 u}-1}$ & 561.36 & $580^{a}$ & 663.97 & $\frac{\text { amu }}{9.046}$ & $\begin{array}{c}\text { amu } \\
58.309\end{array}$ \\
\hline $\mathrm{T}_{1 \mathrm{u}-2}$ & 396.18 & $429^{a}$ & 521.71 & 50.534 & 8.208 \\
\hline $\mathrm{T}_{1 \mathrm{u}}-3$ & 332.18 & $342^{a}$ & 338.81 & 6.714 & 0.200 \\
\hline $\mathrm{T}_{1 \mathrm{u}}-4$ & 174.00 & $175^{a}$ & 175.54 & 0.481 & 0.057 \\
\hline & $\begin{array}{c}\omega_{\mathrm{RA}} \\
\left(\mathrm{cm}^{-1}\right)\end{array}$ & $\begin{array}{c}\omega_{\mathrm{RA}} \\
\left(\mathrm{cm}^{-1}\right)\end{array}$ & $\begin{array}{c}\omega_{\mathrm{RA}} \\
\left(\mathrm{cm}^{-1}\right)\end{array}$ & $\begin{array}{c}\omega_{\mathrm{RA}} \\
\left(\mathrm{cm}^{-1}\right)\end{array}$ & $\begin{array}{c}S_{\mathrm{RA}} \\
\left(\AA^{4} / \mathrm{amu}\right)\end{array}$ \\
\hline$\overline{\mathrm{A}_{1 \mathrm{~g}}}$ & 705.88 & $717^{a}$ & $714^{b}$ & $706^{c}$ & 50.77 \\
\hline $\mathrm{E}_{\mathrm{g}}$ & 368.66 & $395^{a}$ & $-b$ & $-{ }^{c}$ & 0.0651 \\
\hline $\mathrm{T}_{2 \mathrm{~g}-1}$ & 600.89 & $618^{a}$ & $611^{b}$ & $606^{c}$ & 4.784 \\
\hline $\mathrm{T}_{2 \mathrm{~g}-2}$ & 468.21 & $488^{a}$ & $467^{b}$ & $462^{c}$ & 0.5469 \\
\hline$T_{2 g}-3$ & 183.43 & $186^{a}$ & $-{ }^{b}$ & $-c$ & 0.0115 \\
\hline
\end{tabular}

experiment are slightly shifted up in wavenumber with respect to our findings from DFT calculations; however, the agreement is still considered excellent. Additionally, contributions due to impurity modes similar to those previously described by Kasic et al. ${ }^{33}$ for single-crystalline $\mathrm{GaN}$ and Schöche et $a l .{ }^{34}$ for $\mathrm{TiO}_{2}$ were detected in our experimental data. We report five such modes. Their contributions to the dielectric function are best seen in Fig. 7, where we depict the same data shown in Fig. 6 but in a logarithmic scale for the imaginary parts of $\varepsilon$ and its inverse, $\varepsilon^{-1}$. We note that the peaks within the former identify TO modes, while within the latter identify LO modes. This can be clearly observed in Fig. 6 (a) and (b), respectively, and labelled accordingly. Impurity modes appear as peaks with much smaller amplitude, and with frequency identical between the two spectra shown. This can be observed by contributions identified with vertical green dotted lines, labelled accordingly. We note that none of the observed impurity modes fall within the vicinity of our DFT derived Raman-active or silent modes, and their origins cannot be ruled out as being due to elemental impurities or structural defects. Presently, the exact origins are unknown. We note an increase in noise towards the lower as well as towards the higher wavenumber region in Fig. 7, and which is due to the fact that the samples become transparent outside the phonon mode regions. In regions where a sample is transparent, the intensity of light reflected from the samples reduces and thereby increases the error bars on our ellipsometry data. This can be verified with the increase in noise in the same spectral regions in Fig. 2. However, this uncertainty affects the accuracy little within which the phonon and impurity modes can be determined across the phonon mode spectral region.

In Tabl. II, $\varepsilon_{\infty}$ was obtained as a best-match mode dielectric function parameter to be $3.78 \pm 0.02$, and $\varepsilon_{\mathrm{DC}}$ of $10.5 \pm 0.06$ was then obtained using the LST relation. RF capacitance measurements enabled us to directly determine the static di- 

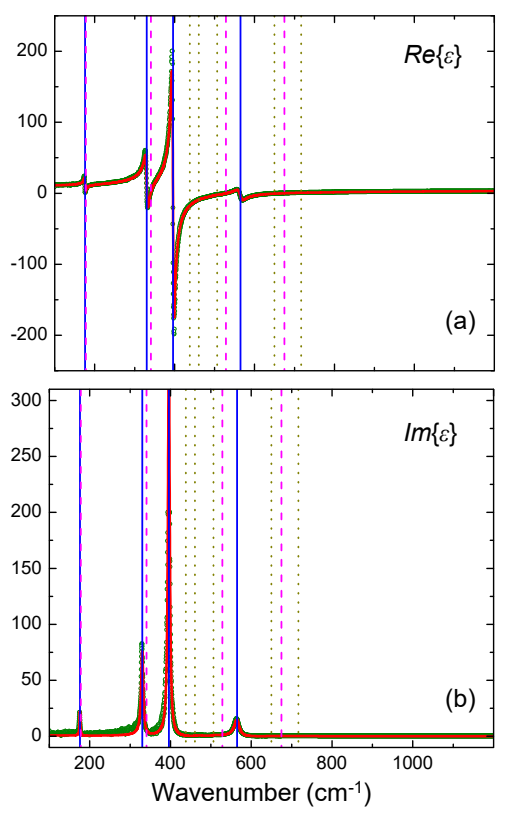

FIG. 6. Wavenumber-by-wavenumber best-match model calculated (green open symbols) and best-match model dielectric function calculated (red solid line) real (a) and imaginary (b) parts of the dielectric function. Vertical blue solid lines indicate TO modes, magenta dashed lines indicate LO modes, and gold dotted lines indicate impurity modes identified in this work.

TABLE II. FPSQ model parameters for TO, LO, and impurity modes obtained from best-match model dielectric function analysis. $\varepsilon_{\infty}$ and $\varepsilon_{\mathrm{DC}}$ are determined as $3.78 \pm 0.02$, and $10.5 \pm 0.06$ using the LST relationship.

\begin{tabular}{ccccccc}
\hline \hline Mode & $\begin{array}{c}\omega_{\mathrm{TO}} \\
\left(\mathrm{cm}^{-1}\right)\end{array}$ & $\begin{array}{c}\omega_{\mathrm{TO}} \\
\left(\mathrm{cm}^{-1}\right)\end{array}$ & $\begin{array}{c}\omega_{\mathrm{TO}} \\
\left(\mathrm{cm}^{-1}\right)\end{array}$ & $\begin{array}{c}\omega_{\mathrm{LO}} \\
\left(\mathrm{cm}^{-1}\right)\end{array}$ & $\begin{array}{c}\gamma_{\mathrm{TO}} \\
\left(\mathrm{cm}^{-1}\right)\end{array}$ & $\begin{array}{c}\gamma_{\mathrm{LO}} \\
\left(\mathrm{cm}^{-1}\right)\end{array}$ \\
\hline
\end{tabular}
$14.9 \pm 0.16 \quad 5.3 \pm 1.6$ itations by phonon scattering at room temperature comparable $\mathrm{T}_{1 \mathrm{u}}-2 \quad 395.99 \pm 0.04 \quad 420^{a} \quad 455^{b} \quad 528.2 \pm 0.45 \quad 5.28 \pm 0.06 \quad 16.3 \pm 0.42$ to those in $\beta-\mathrm{Ga}_{2} \mathrm{O}_{3} .{ }^{47}$ Thus, in addition to the attractiveness

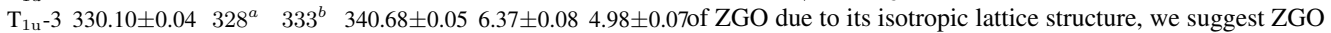

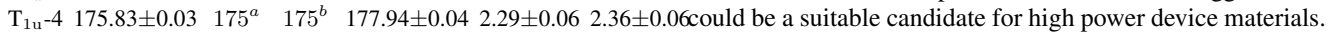
$\mathrm{I}-1 \quad 716 \pm 8.5 \quad 726 \pm 10 \quad 76 \pm 17.2 \quad 76 \pm 13.8$ In summary, we have measured mid-infrared and far-

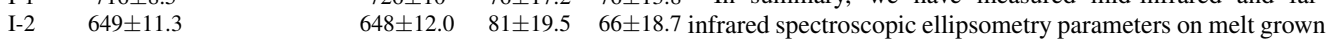
I-3 $506 \pm 1.2 \quad 505 \pm 1.5 \quad 13 \pm 2.4 \quad 11 \pm 2.1$ high quality single bulk crystals of ZGO. We obtained the

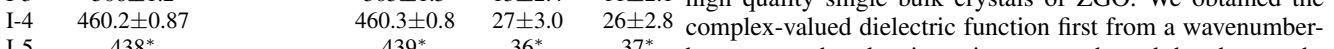

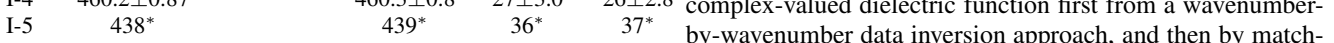
${ }^{a}$ Exp. (IR absorption), Ref. 23. ${ }^{b}$ Exp. (IR absorption), Ref. 28. * Parameter manually held in fit.

electric constant for a similar, insulating sample and resulted in a $\varepsilon_{\mathrm{DC}}$ of $11.3 \pm 0.7$. This value is slightly higher than $\varepsilon_{\mathrm{DC}}$ extrapolated using the LST relationship, and consistent within uncertainty limits with the DFT value. We note that both values are almost in agreement within uncertainty limits, while yet unaccounted for possible defect-activated resonances at longer wavelength would further raise the extrapo- one canticipate for ZGO intrinsic free charge mobility lim-
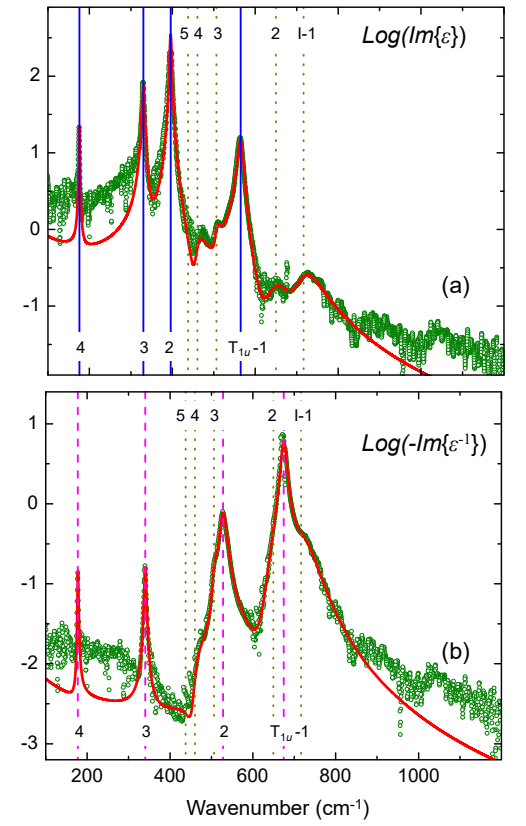

FIG. 7. Same data and line styles as shown in Fig.6, where (a) shows the imaginary part of $\varepsilon$ and (b) shows the imaginary part of its inverse. Note the logarithmic scales for the ordinates.

lated value using the LST relationship. The LST relationship provides the ratio between $\varepsilon_{\mathrm{DC}}$ and $\varepsilon_{\infty}$ and which is a measure for the amount of charge displacement stored within the optical phonons of a polar lattice. We find this ratio similar for spinel ZGO and for $\beta-\mathrm{Ga}_{2} \mathrm{O}_{3}$ when for the latter anisotropic values are averaged over the monoclinic crystal directions. ${ }^{46}$ Also, $\varepsilon_{\mathrm{DC}}$ are similar between the two compounds. Hence, ing a four-parameter semi-quantum model dielectric function model. We observe all four symmetry predicted IR-active lattice modes, split into TO and LO mode pairs. All four IRactive TO and LO modes are in excellent agreement with results from our DFT computations. With the LST relationship we determine the static and high frequency dielectric constants. We further identify five impurity mode pair contributions, whose physical origin remain unknown so far. We provide all Brillouin zone center Raman and silent mode parameters. Our results compare well with incomplete sets of mode parameters obtained previously from polycrystalline samples and DFT studies. 
This work was supported in part by the National Science Foundation under award DMR 1808715, by Air Force Office of Scientific Research under award FA9550-18-1-0360, by the Nebraska Materials Research Science and Engineering Center under award DMR 1420645, the Swedish Research Council VR award No. 2016-00889, the Swedish Foundation for Strategic Research Grant Nos. RIF14-055 and EM16-0024, by the Swedish Governmental Agency for Innovation Systems VINNOVA under the Competence Center Program Grant No. 2016-05190, by the Knut and Alice Wallenbergs Foundation supported grant 'Wide-bandgap semiconductors for next generation quantum components', and by the Swedish Government Strategic Research Area in Materials Science on Functional Materials at Linköping University, Faculty Grant SFO Mat LiU No. 2009-00971. M. S. acknowledges the University of Nebraska Foundation and the J. A. Woollam Foundation for financial support.

The data that support the findings of this study are available from the corresponding author upon reasonable request.

${ }^{1}$ M. Grundmann, H. Frenzel, A. Lajn, M. Lorenz, F. Schein, and H. von Wenckstern, phys. stat. sol. (a) 207, 1437 (2010).

${ }^{2}$ M. Lorenz, M. S. R. Rao, T. Venkatesan, E. Fortunato, P. Barquinha, R. Branquinho, D. Salgueiro, R. Martins, E. Carlos, A. Liu, F. K. Shan, M. Grundmann, H. Boschker, J. Mukherjee, M. Priyadarshini, N. DasGupta, D. J. Rogers, F. H. Teherani, E. V. Sandana, P. Bove, K. Rietwyk, A. Zaban, A. Veziridis, A. Weidenkaff, M. Muralidhar, M. Murakami, S. Abel, J. Fompeyrine, J. Zuniga-Perez, R. Ramesh, N. A. Spaldin, S. Ostanin, V. Borisov, I. Mertig, V. Lazenka, G. Srinivasan, W. Prellier, M. Uchida, M. Kawasaki, R. Pentcheva, P. Gegenwart, F. M. Granozio, J. Fontcuberta, and N. Pryds, J. Phys. D: Appl. Phys. 49, 433001 (2016). ${ }^{3}$ S. J. Pearton, J. Yang, P. H. Cary, F. Ren, J. Kim, M. J. Tadjer, and M. A. Mastro, Appl. Phys. Rev. 5, 011301 (2018).

${ }^{4}$ Z. Galazka, S. Ganschow, R. Schewski, K. Irmscher, D. Klimm, A. Kwasniewski, M. Pietsch, A. Fiedler, I. Schulze-Jonack, M. Albrecht, T. Schröder, and M. Bickermann, APL Mater. 7, 022512 (2019).

${ }^{5}$ Z. Galazka, D. Klimm, K. Irmscher, R. Uecker, M. Pietsch, R. Bertram, M. Naumann, M. Albrecht, A. Kwasniewski, R. Schewski, and M. Bickermann, phys. stat. sol. (a) 212, 1455 (2015)

${ }^{6}$ A. Mock, R. Korlacki, C. Briley, V. Darakchieva, B. Monemar, Y. Kumagai, K. Goto, M. Higashiwaki, and M. Schubert, Phys. Rev. B 96, 245205 (2017).

${ }^{7}$ Z Galazka, Semicond. Sci. Technol. 33, 113001 (2018).

${ }^{8}$ M. Higashiwaki, K. Sasaki, A. Kuramata, T. Masui, and S. Yamakoshi, phys. stat. sol. (a) 211, 2126 (2014)

${ }^{9}$ M. Higashiwaki and G. H. Jessen, Appl. Phys. Lett. 112, 060401 (2018).

${ }^{10}$ Z. Xia, H. Chandrasekar, W. Moore, C. Wang, A. J. Lee, J. McGlone, N. K. Kalarickal, A. Arehart, S. Ringel, F. Yang, and S. Rajan, Appl. Phys. Lett. 115, 252104 (2019).

${ }^{11}$ J. Zhang, J. Shi, D.-C. Qi, L. Chen, and K. H. L. Zhang, APL Materials 8 , 020906 (2020).

${ }^{12}$ T. J. Flack, B. N. Pushpakaran, and S. B. Bayne, J. Electron. Mater. 45 , 2673 (2016)

${ }^{13}$ A. Bessière, S. Jacquart, K. Priolkar, A. Lecointre, B. Viana, and D. Gourier, Opt. Express 19, 10131 (2011).

${ }^{14}$ I. J. Hsieh, K. T. Chu, C. F. Yu, and M. S. Feng, J. Appl. Phys., 3735 (1994).

${ }^{15}$ S. Yan, S. Ouyang, J. Gao, M. Yang, J. Feng, X. Fan, L. Wan, Z. Li, J. Ye, Y. Zhou, and Z. Zou, Angew. Chem. Int. Ed. 49, 6400 (2010).

${ }^{16} \mathrm{Z}$. Yan and H. Takei, J. Cryst. Growth 171, 131 (1997).

${ }^{17}$ P. J. Van der Straten, R. Metselaar, and H. D. Jonker, J. Cryst. Growth 43, 270 (1978)
${ }^{18}$ A. B. Chase and J. A. Osmer, J. Am. Ceram. Soc. 50, 325 (1967).

${ }^{19}$ S. H. Tsai, S. Basu, C. Y. Huang, L. C. Hsu, Y. G. Lin, and R. H. Horng, Sci. Rep. 8, 14056 (2018).

${ }^{20}$ S. K. Sampath, D. G. Kanhere, and R. Pandey, J. Phys.: Cond. Mat. 11, 3635 (1999).

${ }^{21}$ W. K. Wang, K. F. Liu, P. C. Tsai, Y. J. Xu, and S. Y. Huang, Coatings 9 , 859 (2019).

${ }^{22}$ W. K. Wang, Y. J. Xu, S. Y. Huang, K. F. Liu, and P. C. Tsai, Coatings 9 , 469 (2019).

${ }^{23}$ G. G. Van Gorkom, J. H. Haanstra, and H. v. d. Boom, J. Raman Spectrosc. 1, 513 (1973).

${ }^{24}$ G. G. Van Gorkom, J. C. Henning, and R. P. Van Stapele, Phys. Rev. B 8 955 (1973).

${ }^{25}$ J. E. Drake and J. Simpson, Spectrochim. Acta Part A Mol. Spectrosc. 24, 981 (1968).

${ }^{26}$ H. A. Lauwers and M. A. Herman, J. Phys. Chem. Solids 41, 223 (1980).

${ }^{27}$ S. López, A. H. Romero, P. Rodríguez-Hernández, and A. Muñoz, Phys. Rev. B 79, 214103 (2009).

${ }^{28} \mathrm{~J}$. Preudhomme and P. Tarte, Spectrochim. Acta Part A Mol. Spectrosc. 27, 1817 (1971).

${ }^{29}$ S. López-Moreno, P. Rodríguez-Hernández, A. Muñoz, A. H. Romero, F. J. Manjón, D. Errandonea, E. Rusu, and V. V. Ursaki, Ann. der Phys. 523 157 (2011).

${ }^{30}$ P. Kühne, C. M. Herzinger, M. Schubert, J. A. Woollam, and T. Hofmann, Rev. Sci. Instrum. 85, 071301 (2014).

${ }^{31} \mathrm{M}$. Schubert, Infrared Ellipsometry on Semiconductor Layer Structures: Phonons, Plasmons and Polaritons, Springer Tracts in Modern Physics, Vol. 209 (Springer, Berlin, 2004)

${ }^{32}$ F. Gervais and B. Piriou, J. Phys. C: Solid State Physics 7, 2374 (1974).

${ }^{33}$ A. Kasic, M. Schubert, S. Einfeldt, D. Hommel, and T. E. Tiwald, Phys. Rev. B 62, 7365 (2000).

${ }^{34}$ S. Schöche, T. Hofmann, R. Korlacki, T. E. Tiwald, and M. Schubert, J. Appl. Phys. 113, 111906 (2013)

${ }^{35}$ R. H. Lyddane, R. Sachs, and E. Teller, Phys. Rev. 59, 613 (1941).

${ }^{36}$ Quantum ESPRESSO is available from http://www.quantum-espresso.org. See also: P. Giannozzi, S. Baroni, N. Bonini, M. Calandra, R. Car C. Cavazzoni, D. Ceresoli, G. L. Chiarotti, M. Cococcioni, I. Dabo, A. D Corso, S. de Gironcoli, S. Fabris, G. Fratesi, R. Gebauer, U. Gerstmann, C. Gougoussis, A. Kokalj, M. Lazzeri, L. Martin-Samos, N. Marzari, F. Mauri, R. Mazzarello, S. Paolini, A. Pasquarello, L. Paulatto, C. Sbraccia, S. Scandolo, G. Sclauzero, A. P. Seitsonen, A. Smogunov, P. Umari, and R. M. Wentzcovitch, J. Phys.: Cond. Mat. 21, 395502 (2009).

${ }^{37}$ J. P. Perdew and Y. Wang, Phys. Rev. B 45, 13244 (1992).

${ }^{38}$ J. P. Perdew and Y. Wang, Phys. Rev. B 98, 079904 (2018)

${ }^{39}$ M. J. van Setten, M. Giantomassi, E. Bousquet, M. J. Verstraete, D. R Hamann, X. Gonze, and G. M. Rignanese, Comput. Phys. Commun. 226, 39 (2018), 1710.10138 .

${ }^{40}$ A. Jain, S. P. Ong, G. Hautier, W. Chen, W. D. Richards, S. Dacek, S. Cholia, D. Gunter, D. Skinner, G. Ceder, and K. A. Persson, APL Mat. 1, 011002 (2013)

${ }^{41}$ K. Persson, DoE Data Explorer mp-5794, SG:227 (2014).

${ }^{42}$ H. J. Monkhorst and J. D. Pack, Phys. Rev. B 13, 5188 (1976).

${ }^{43}$ P. L. Polavarapu, J. Phys. Chem. 94, 8106 (1990).

${ }^{44}$ S. Baroni, S. de Gironcoli, A. D. Corso, S. Baroni, S. de Gironcoli, and P. Giannozzi, Rev. Mod. Phys. 73, 515 (2001).

${ }^{45}$ A. Kokalj, J. Mol. Graph. Model. 17, 176 (1999). Code available from http://www.xcrysden.org.

${ }^{46}$ M. Schubert, R. Korlacki, S. Knight, T. Hofmann, S. Schöche, V. Darakchieva, E. Janzén, B. Monemar, D. Gogova, Q.-T. Thieu, R. Togashi, H. Murakami, Y. Kumagai, K. Goto, A. Kuramata, S. Yamakoshi, and M. Higashiwaki, Phys. Rev. B 93, 125209 (2016).

${ }^{47} \mathrm{~N}$. Ma, N. Tanen, A. Verma, Z. Guo, T. Luo, H. G. Xing, and D. Jena, Applied Physics Letters 109, 212101 (2016) https://doi.org/10.1063/1.4968550. 


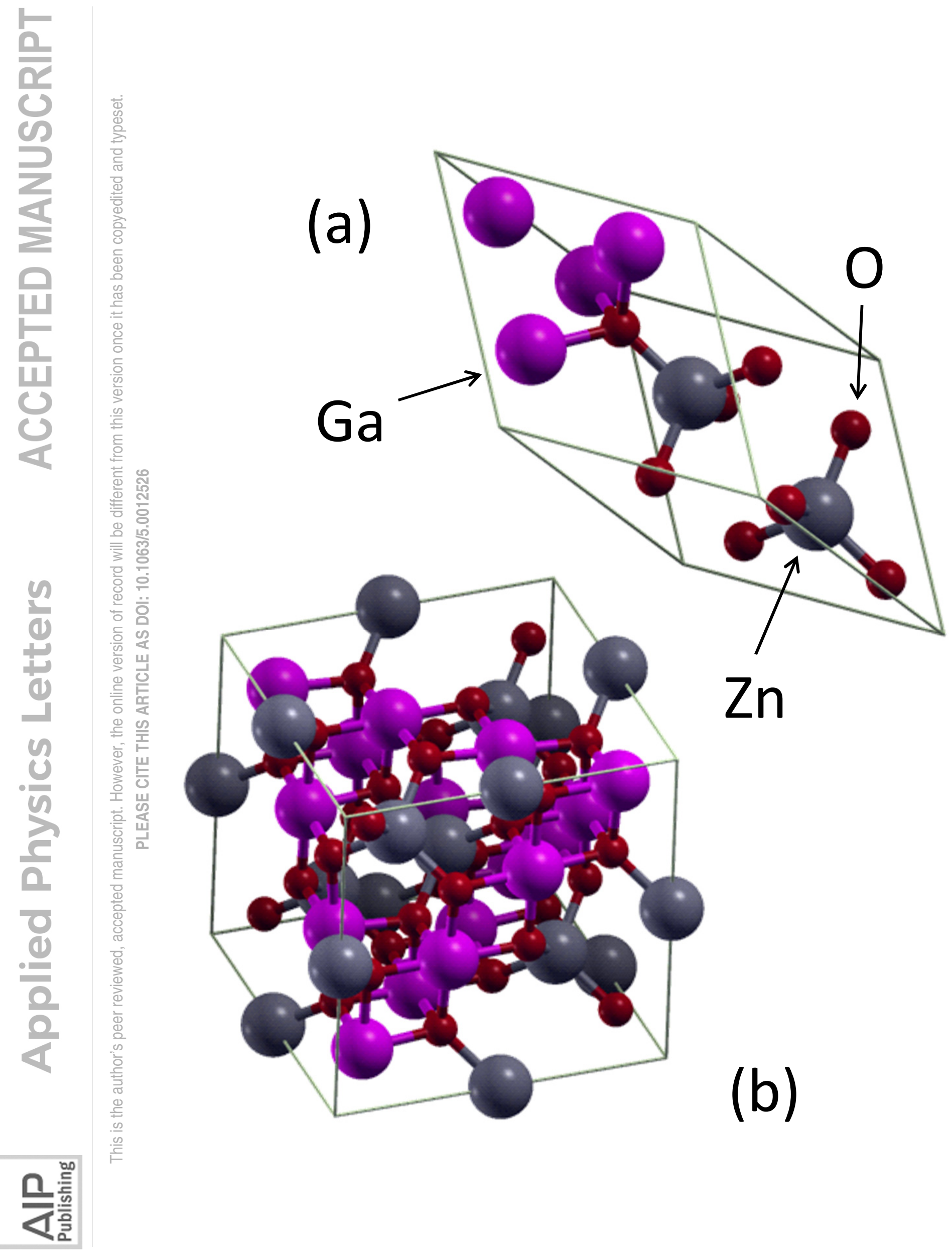



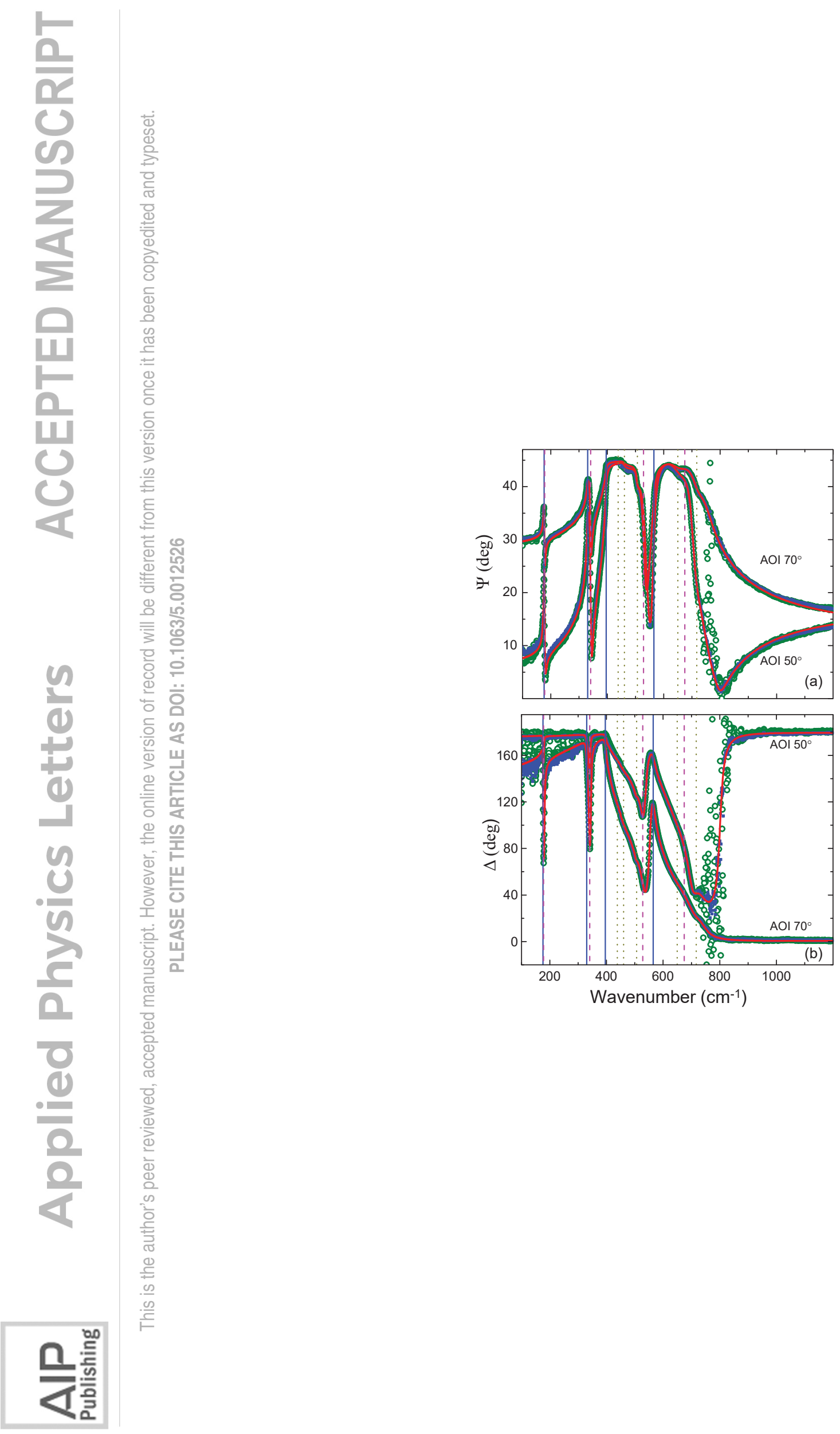

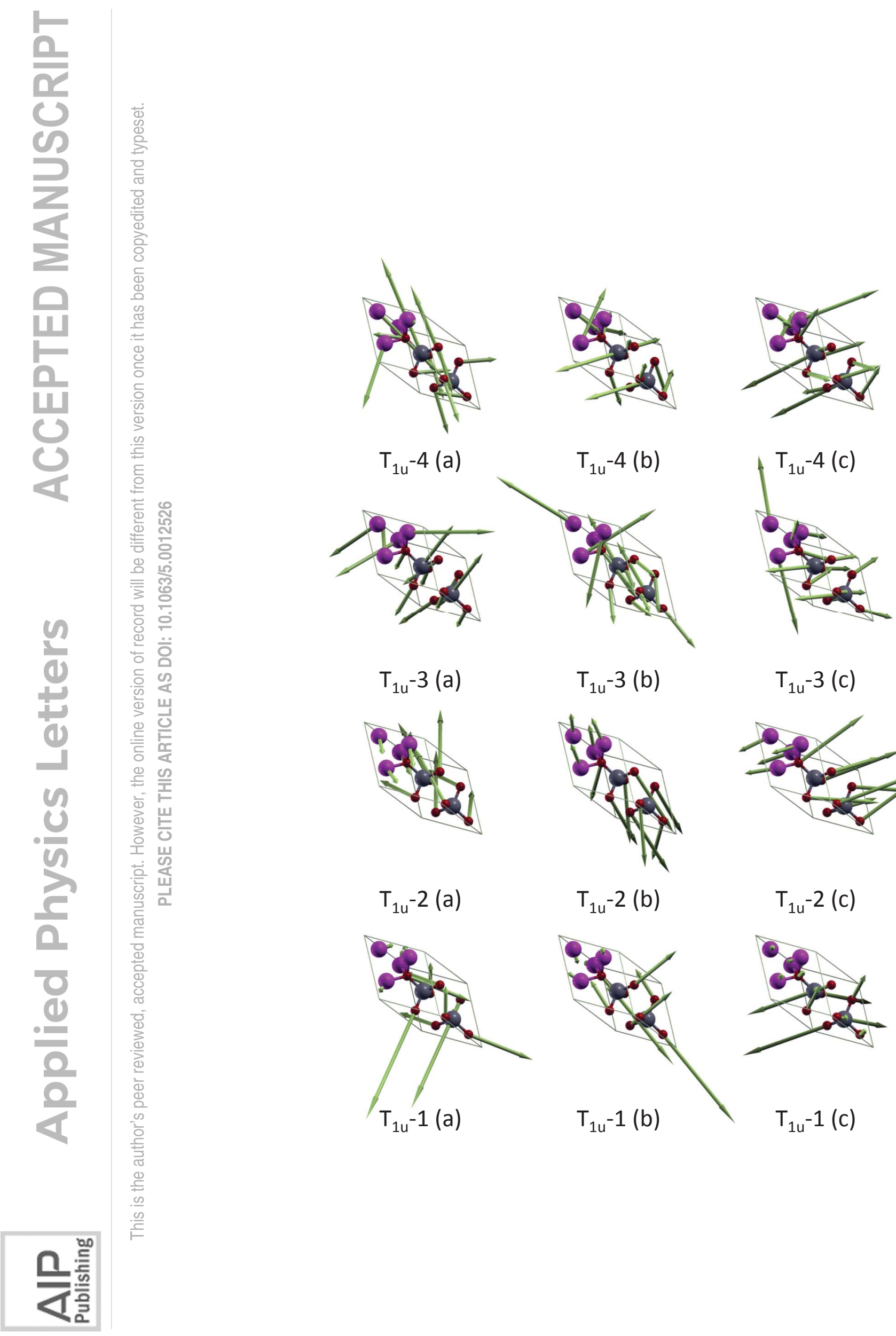

$$
\mathrm{T}_{1 \mathrm{u}}-2 \text { (b) }
$$
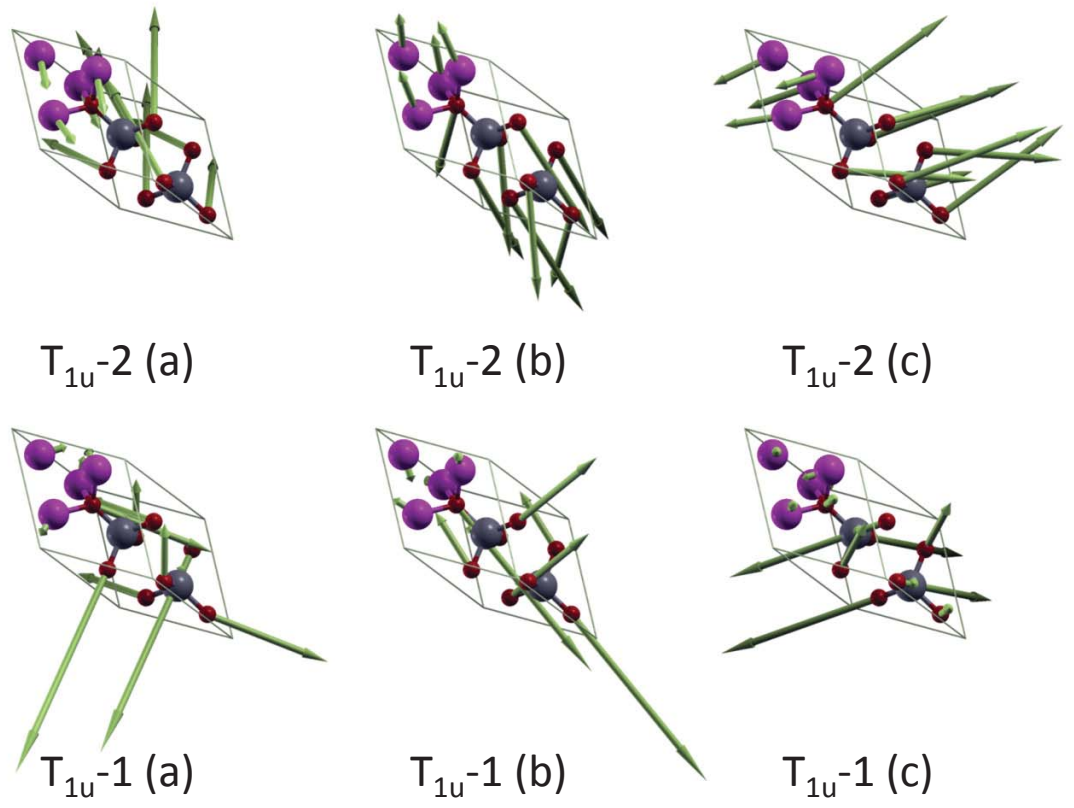
$n$

(1)

1

(1)

1
0
1
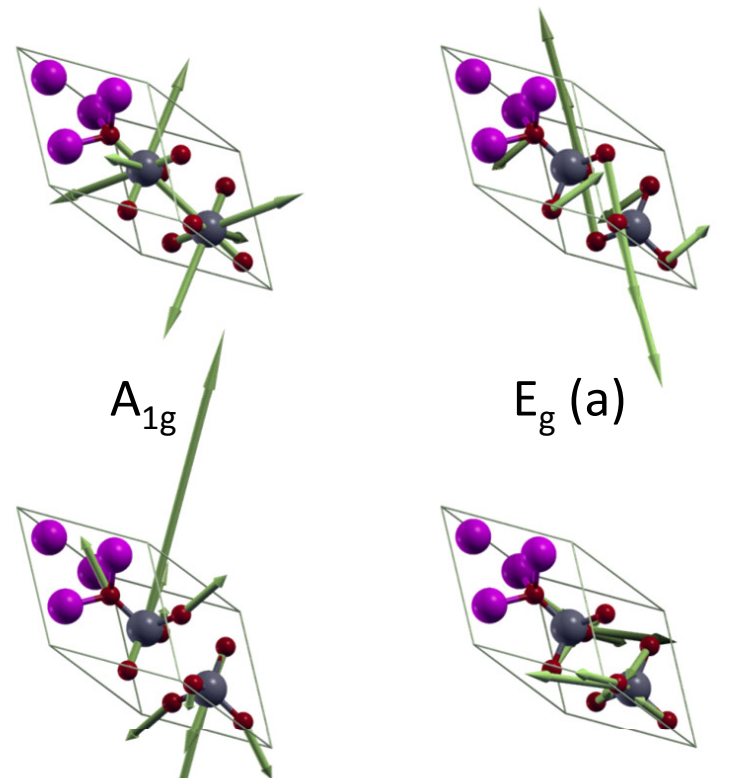

$E_{g}(a)$
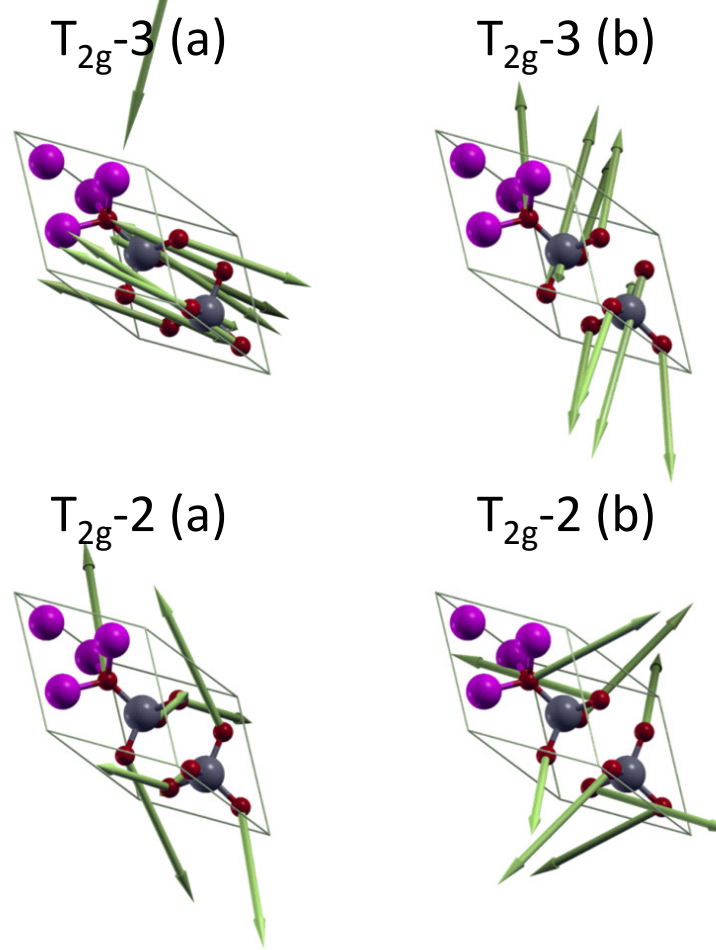

$\mathrm{T}_{2 \mathrm{~g}}-1$ (a)
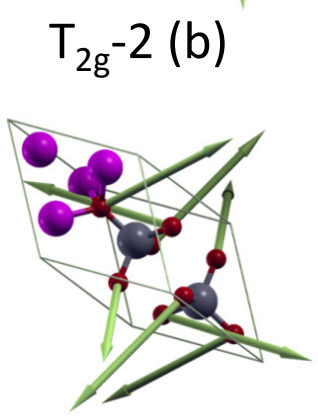

$T_{2 g}-1$ (b)

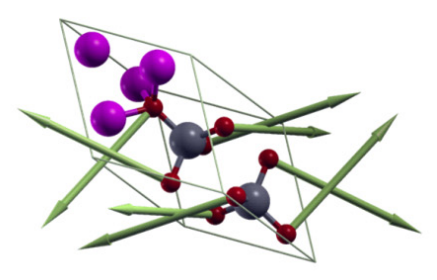

$E_{g}(b)$

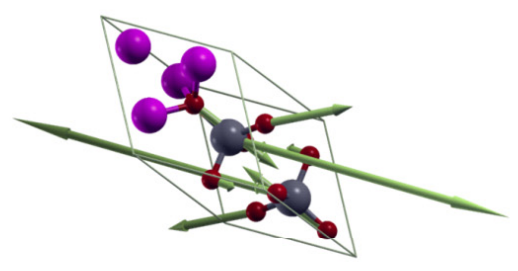

$T_{2 g}-3(c)$

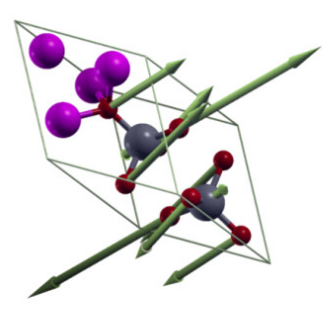

$T_{2 g}-2$ (c)

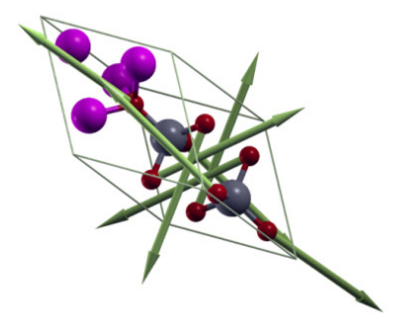

$T_{2 g}-1(c)$ 

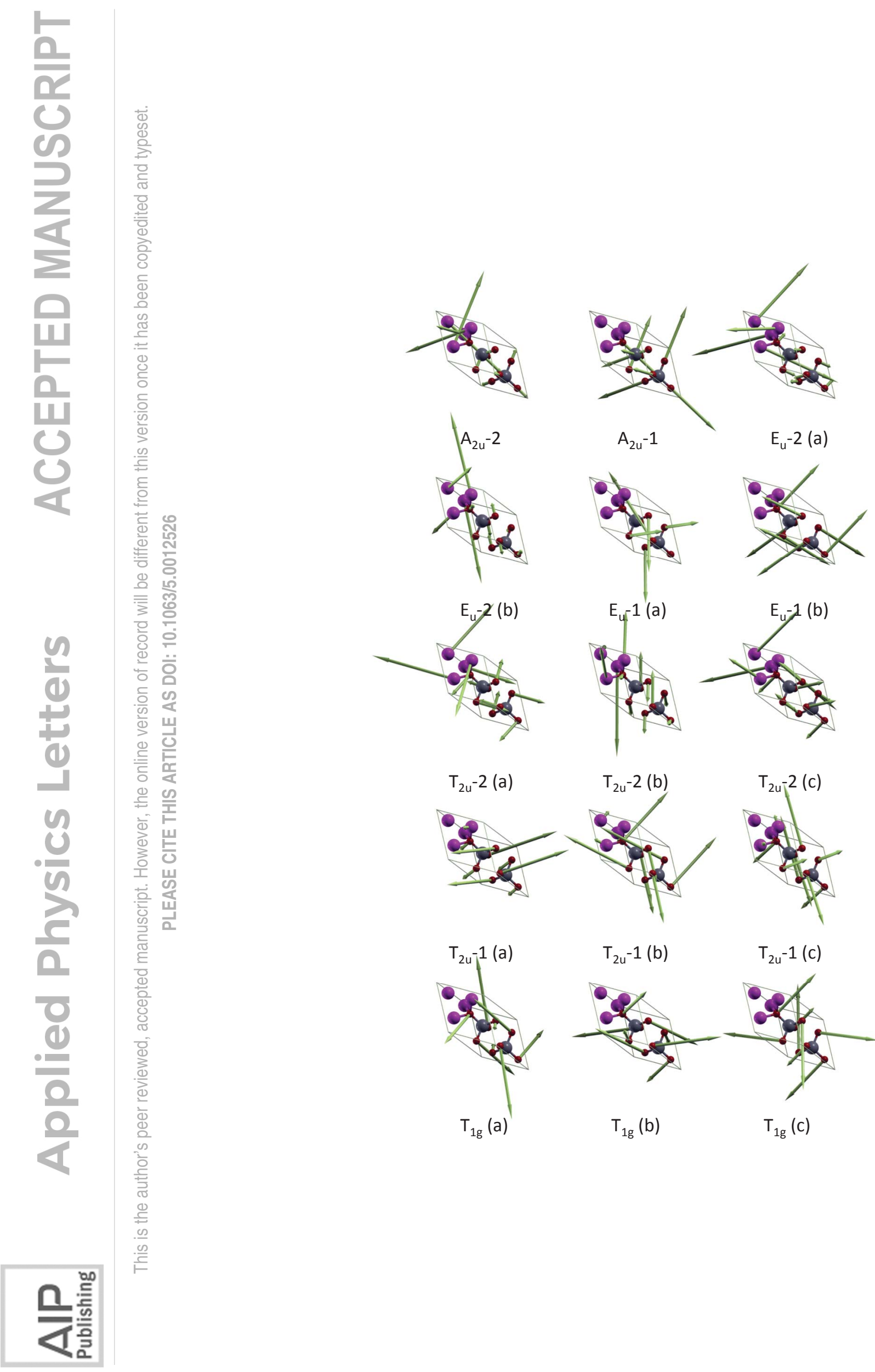

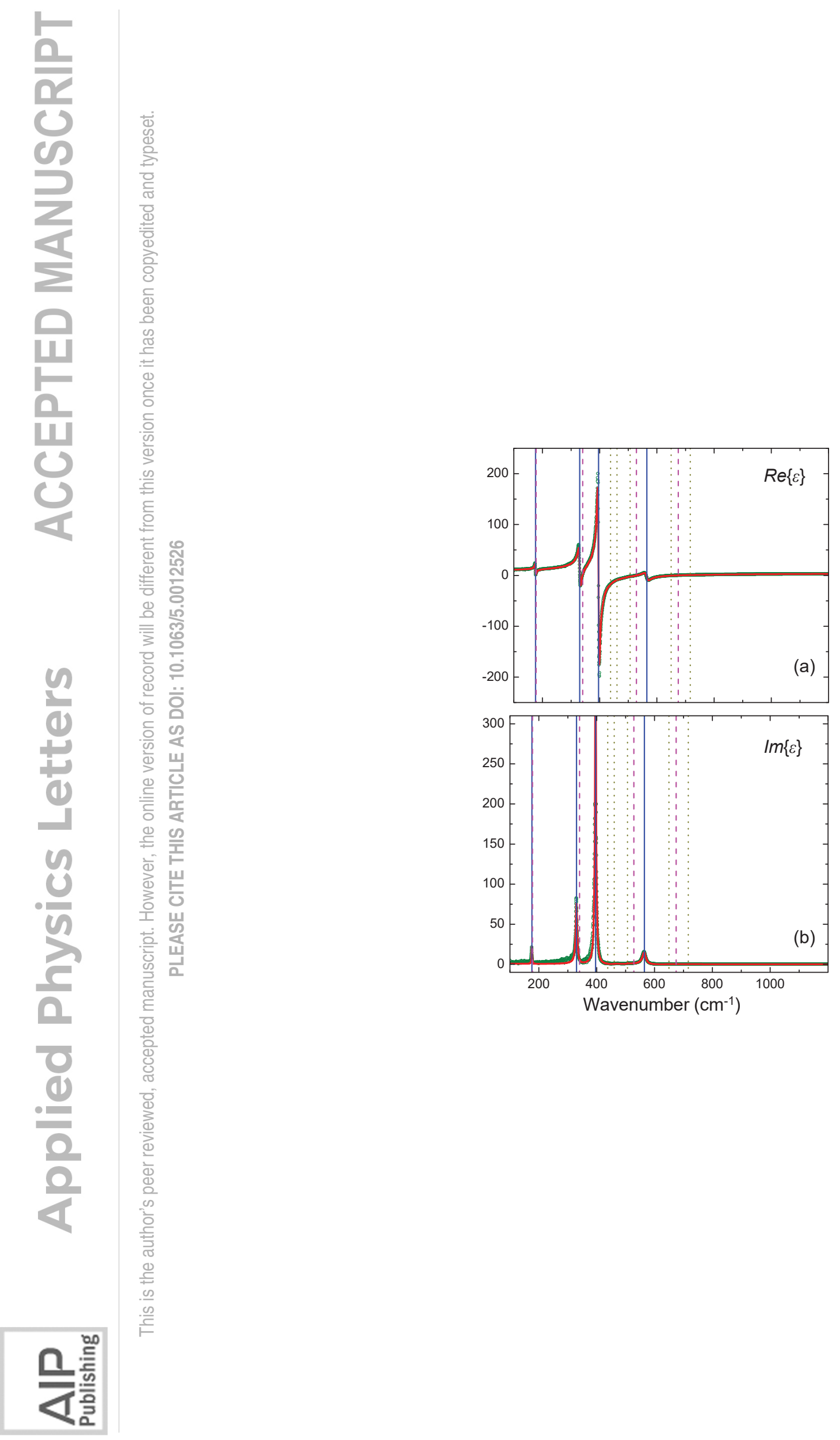

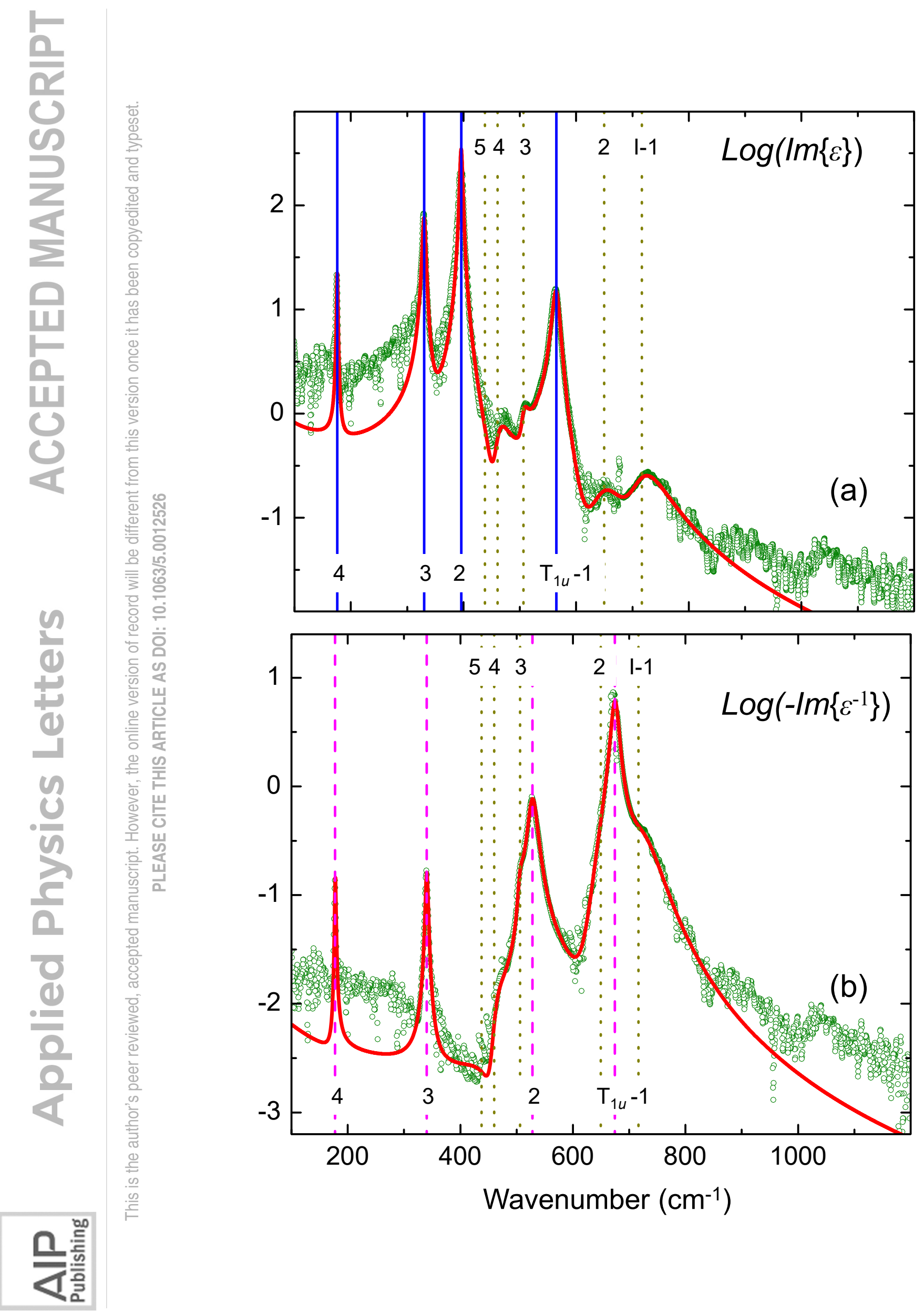